PROCEEDINGS OF THE

AMERICAN MATHEMATICAL SOCIETY

Volume 125, Number 1, January 1997, Pages 199-202

S 0002-9939(97)03647-2

\title{
A NOTE ON THE KOBAYASHI-ROYDEN METRIC FOR REAL ELLIPSOIDS
}

\author{
WŁODZIMIERZ ZWONEK
}

(Communicated by Eric Bedford)

\begin{abstract}
We give a proof of the fact proven by L.D. Kay that the KobayashiRoyden metric of a real ellipsoid (of dimension at least 2) at 0 is hermitian exactly when the ellipsoid is the ball. The proof given by us is much simpler and shorter than that of Kay although it is based on the same results.
\end{abstract}

Denote by $E$ the unit disk in $\mathbb{C}$. For a domain $D \subset \mathbb{C}^{n}$ we put for $(z, X) \in D \times \mathbb{C}^{n}$

$$
\kappa_{D}(z ; X):=\inf \left\{\alpha>0: \text { there is } \varphi: E \rightarrow D \text { with } \varphi(0)=z, \alpha \varphi^{\prime}(0)=X\right\} .
$$

$\kappa_{D}$ is called the Kobayashi-Royden pseudometric.

In case $D$ is a strongly convex domain we get from Lempert's theorem (see [Lem]) the existence and uniqueness of the mappings attaining infimum in the definition of $\kappa_{D}(z ; X)$ (with $X \neq 0$ ). Those mappings are called extremal.

For the basic properties of $\kappa_{D}$ see [Jar-Pfl]. Let us mention here only one property, namely, the homogeneity of the Kobayashi-Royden pseudometric with respect to the second variable, i.e.

$$
\kappa_{D}(z, \lambda X)=|\lambda| \kappa_{D}(z, X), \quad(z, X) \in D \times \mathbb{C}^{n}, \lambda \in \mathbb{C} .
$$

For fixed $\mu=\left(\mu_{1}, \ldots, \mu_{n}\right) \in[0,1)^{n}$, where $n \in \mathbb{N}_{*}$, define

$$
\mathcal{E}(\mu):=\left\{\left(z_{1}, \ldots, z_{n}\right) \in \mathbb{C}^{n}: \sum_{j=1}^{n}\left(\left|z_{j}\right|^{2}+\mu_{j} \operatorname{Re} z_{j}^{2}\right)<1\right\} .
$$

The domains $\mathcal{E}(\mu)$ as in $(1)$ are called real ellipsoids.

Below let us recall some basic properties of real ellipsoids.

Remark 1. (a) A real ellipsoid is always a strongly convex bounded domain with analytic boundary. Moreover, the unit outer vector $\nu(z)$ to $\partial \mathcal{E}(\mu)$ at $z \in \partial \mathcal{E}(\mu)$ equals $p(z)\left(z_{1}+\mu_{1} \overline{z_{1}}, \ldots, z_{n}+\mu_{n} \overline{z_{n}}\right)$, where $p(z)>0$;

(b) $\kappa_{\mathcal{E}(\mu)}\left(0 ;\left( \pm v_{1}, \ldots, \pm v_{n}\right)\right)=\kappa_{\mathcal{E}(\mu)}\left(0 ;\left(v_{1}, \ldots, v_{n}\right)\right)$ for any $\left(v_{1}, \ldots, v_{n}\right) \in \mathbb{C}^{n}$.

Below we shall prove the following theorem:

Theorem 2 (cf. [Kay]). Let $\mathcal{E}$ be a real ellipsoid in $\mathbb{C}^{n}, n \geq 2$. Then $\mathcal{E}$ is biholomorphic to the ball in $\mathbb{C}^{n}$ if and only if its Kobayashi-Royden metric at 0 is hermitian.

Received by the editors July 21, 1995.

1991 Mathematics Subject Classification. Primary 32H15.

This paper has been supported by KBN grant No 2 PO3A 06008.

(C)1997 American Mathematical Society 
The proof given by us is much simpler than the original one; it involves almost no computations although it is based on similar results as the proof in [Kay]. Below we shall omit some parts of the proof that are identical to that presented in [Kay], referring the interested reader to that paper.

For the proof of Theorem 2 we need the following lemma:

Lemma 3. Let $\mathcal{E}(\mu) \subset \mathbb{C}($ so $\mu \in[0,1))$. Then

$$
\kappa_{\mathcal{E}(\mu)}(0 ; 1) \geq 1 \text {. }
$$

Moreover, the equality in (2) holds iff $\mu=0$.

Proof of Lemma 3. As the case $\mu=0$ is trivial, we restrict ourselves only to the case $\mu>0$.

Due to the considerations in [Kay] we have the following formula:

$$
\kappa_{\mathcal{E}(\mu)}(0 ; 1)^{2}=\frac{1-\mu^{2}}{\mu} 2 \sum_{n=1}^{\infty} \rho^{n-\frac{1}{2}} \frac{1+\rho^{2 n-1}}{\left(1-\rho^{2 n-1}\right)^{2}},
$$

where $\rho=\frac{\left(1-\sqrt{1-\mu^{2}}\right)^{2}}{\mu^{2}}$ (see the formula (1.1) and the first formula on the page 60 in $[$ Kay $]$ ).

Using (3) we get

$$
\kappa_{\mathcal{E}(\mu)}(0 ; 1)^{2}>\frac{1-\mu^{2}}{\mu} 2 \rho^{\frac{1}{2}} \frac{1+\rho}{(1-\rho)^{2}}=2\left(1-\mu^{2}\right) \frac{\left(1-\sqrt{1-\mu^{2}}\right)\left(2-2 \sqrt{1-\mu^{2}}\right)}{\left(2 \mu^{2}-2+2 \sqrt{1-\mu^{2}}\right)^{2}}=1 .
$$

This completes the proof of the lemma.

Proof of Theorem 2. Using the reasoning as in [Kay], we see that to prove the theorem it is enough to disprove the parallelogram law in the two-dimensional case with $\mu_{1}^{2}+\mu_{2}^{2}>0$. To get a contradiction suppose that the parallelogram law (for simplicity we write $\left.\kappa(v):=\kappa_{\mathcal{E}\left(\mu_{1}, \mu_{2}\right)}((0,0) ; v)\right)$

$$
2\left(\kappa(u)^{2}+\kappa(v)^{2}\right)=\kappa(u+v)^{2}+\kappa(u-v)^{2}
$$

holds for any $u, v \in \mathbb{C}^{2}$.

Take also $s>0$ such that

$$
s \kappa(1,0)=s \kappa_{\mathcal{E}\left(\mu_{1}\right)}(0 ; 1)=\kappa(0,1)=\kappa_{\mathcal{E}\left(\mu_{2}\right)}(0 ; 1) .
$$

Below we consider two cases.

Case (I). $\mu_{1}, \mu_{2}>0$.

In this case we define the following holomorphic mappings:

$$
\begin{gathered}
\varphi: E \ni \lambda \rightarrow \frac{1}{\sqrt{1+\mu_{1} / \mu_{2}}}\left(\lambda, i \sqrt{\mu_{1} / \mu_{2}} \lambda\right) \in \mathcal{E}\left(\mu_{1}, \mu_{2}\right), \\
\left.\psi: E \ni \lambda \rightarrow \frac{1}{\sqrt{1+\mu_{2} / \mu_{1}}}\left(i \sqrt{\mu_{2} / \mu_{1}} \lambda, \lambda\right)\right) \in \mathcal{E}\left(\mu_{1}, \mu_{2}\right) .
\end{gathered}
$$

In view of the definition of the Kobayashi-Royden metric we get

$$
\begin{gathered}
\kappa\left(1, i \sqrt{\mu_{1} / \mu_{2}}\right) \leq \sqrt{1+\mu_{1} / \mu_{2}}, \\
\kappa\left(i \sqrt{\mu_{2} / \mu_{1}}, 1\right) \leq \sqrt{1+\mu_{2} / \mu_{1}}
\end{gathered}
$$

(in fact one may even prove that in (8) and (9) we have the equalities). 
In view of Remark 1(b), Lemma 3, (4), (5) and the homogeneity of the Kobayashi-Royden metric we get

$$
\begin{aligned}
& 1+\mu_{1} / \mu_{2} \geq \kappa\left(1, i \sqrt{\mu_{1} / \mu_{2}}\right)^{2}=\kappa(1,0)^{2}+\kappa\left(0, i \sqrt{\mu_{1} / \mu_{2}}\right)^{2}= \\
& \kappa(1,0)^{2}+\mu_{1} / \mu_{2} \kappa(0,1)^{2}=\left(1+\mu_{1} / \mu_{2} s^{2}\right) \kappa(1,0)^{2}>1+\mu_{1} / \mu_{2} s^{2} .
\end{aligned}
$$

Analogously, we get

$$
1+\mu_{2} / \mu_{1}>1+\mu_{2} / \mu_{1} \frac{1}{s^{2}} .
$$

From (10) we get $1>s^{2}$, whereas from (11) we get $1>1 /\left(s^{2}\right)$ - a contradiction.

Case (II). $\mu_{1} \mu_{2}=0, \mu_{1}^{2}+\mu_{2}^{2}>0$. Without loss of generality we may assume that $\mu_{1}=0, \mu_{2}>0$.

In view of Remark 1(b), (4) and (5) we get:

$$
\kappa(1,1 / s)^{2}=\kappa(1,0)^{2}+\kappa(0,1 / s)^{2}=2 \kappa(1,0)^{2}=2 .
$$

Let $\psi: E \rightarrow \mathcal{E}\left(\mu_{2}\right)$ be a conformal mapping with $\psi(0)=0, \psi^{\prime}(0)>0$; then, certainly, due to regularity of the boundary of $\mathcal{E}\left(\mu_{2}\right), \psi$ extends continuously to $\bar{E}$ and from (5) we get $\psi^{\prime}(0)=1 / s$.

Define

$$
\varphi: E \ni \lambda \rightarrow \frac{1}{\sqrt{2}}(\lambda, \psi(\lambda)) \in \mathcal{E}\left(0, \mu_{2}\right)
$$

Certainly

$$
\varphi(0)=(0,0), \quad \varphi^{\prime}(0)=\frac{1}{\sqrt{2}}(1,1 / s),
$$

which in view of (12) implies that $\varphi$ is extremal. Consequently, in view of the Lempert's theorem (cf. [Lem] and compare Remark 1(a)) there are functions $h_{1}, h_{2} \in \mathcal{O}(E, \mathbb{C}) \cap \mathcal{C}(\bar{E}, \mathbb{C}), \rho \in \mathcal{C}(\partial E,(0, \infty))$ such that

$$
\begin{gathered}
\frac{1}{\lambda} h_{1}(\lambda)=\rho(\lambda) \frac{1}{\sqrt{2}} \bar{\lambda}, \\
\frac{1}{\lambda} h_{2}(\lambda)=\rho(\lambda) \frac{1}{\sqrt{2}}\left(\overline{\psi(\lambda)}+\mu_{2} \psi(\lambda)\right),
\end{gathered}
$$

for any $\lambda \in \partial E$.

From (13) we get

$$
h_{1}(\lambda)=\frac{1}{\sqrt{2}} \rho(\lambda), \quad \lambda \in \partial E .
$$

Since the right-hand side of the last equality is real, $h_{1}$ is constant on $E$ and then also $\rho(\lambda) \equiv \varepsilon$ for some $\varepsilon>0, \lambda \in \partial E$. Consequently, we get from (14) on $\partial E$ the equality

$$
\frac{1}{\lambda}\left(h_{2}(\lambda) \psi(\lambda)-\frac{1}{\sqrt{2}} \mu_{2} \varepsilon \psi^{2}(\lambda) \lambda\right)=\varepsilon \frac{1}{\sqrt{2}}|\psi(\lambda)|^{2} .
$$

So Gentili's result (see [Gen]) implies that for any $\lambda \in E$

$$
h_{2}(\lambda) \psi(\lambda)-\frac{1}{\sqrt{2}} \mu_{2} \varepsilon \psi^{2}(\lambda) \lambda=r \lambda
$$

where $r>0$ (remember that $\psi(0)=0)$. 
The last equality together with (15) implies that $|\psi(\lambda)| \equiv$ const for $\lambda \in \partial E$, which contradicts, however, the conformality of the mapping $\psi$ between $E$ and $\mathcal{E}\left(\mu_{2}\right)$.

\section{REFERENCES}

[Gen] G. Gentili, Regular complex geodesics in the domain $D_{n}=\left\{\left(z_{1}, \ldots, z_{n}\right) \in \mathbb{C}^{n}:\left|z_{1}\right|+\right.$ $\left.\ldots+\left|z_{n}\right|<1\right\}$, in: Lecture Notes in Math. 1277, Springer, (1987), 35-45. MR 89f:32044

[Jar-Pfl] M. Jarnicki, P. Pflug, Invariant Distances and Metrics in Complex Analysis, Walter de Gruyter, 1993. MR 94k:32039

[Kay] L. D. Kay, On the Kobayashi-Royden metric for ellipsoids, Math. Ann. 289 (1991), 55-72. MR 92a:32030

[Lem] L. Lempert, La métrique de Kobayashi et la représentation des domaines sur la boule, Bull. Soc. Math. Fr. 109 (1981), 427-474. MR 84d:32036

Instytut Matematyki, Uniwersytet Jagielloński, Reymonta 4, 30-059 Kraków, PolAND

E-mail address: zwonek@im.uj.edu.pl 